\section{A Framework for Performance Characterization of Intermediate-Level Grouping Modules}

\author{
Sudhir Borra and Sudeep Sarkar
}

\begin{abstract}
We present five performance measures to evaluate grouping modules in the context of constrained search and indexing based object recognition. Using these measures, we demonstrate a sound experimental framework, based on statistical ANOVA tests, to compare and contrast three edge based organization modules, namely, those of Etemadi et al., Jacobs, and Sarkar-Boyer in the domain of aerial objects using 50 images. With adapted parameters, the Jacobs module performs overall the best for constraint based recognition. For fixed parameters, the Sarkar-Boyer module is the best in terms of recognition accuracy and indexing speedup. Etemadi et al.'s module performs equally well with fixed and adapted parameters while the Jacobs module is most sensitive to fixed and adapted parameter choices. The overall performance ranking of the modules is Jacobs, Sarkar-Boyer, and Etemadi et al.
\end{abstract}

Index Terms-Perceptual organization, performance evaluation, analysis of variance, ANOVA, experimental vision, intermediate level computer vision, feature grouping, performance characterization.

\section{INTRODUCTION}

PERCEPTUAL organization or feature grouping is primarily a bottom-up process which clusters image features that are most likely to come from a single object. Grimson [4] has shown that the combinatorics of the recognition process in cluttered environments using constrained search reduces from an exponential to a low order polynomial if we use an intermediate grouping process which need not be perfect. The importance of grouping is also true for indexing based recognition strategies. Clemens and Jacobs [5] have shown that recognition by "indexing is of very limited benefit unless accompanied by a grouping process."

In response to the importance of low-level feature grouping, various types of perceptual organization modules have been proposed [3], [6], [2], [1]. These modules not only use different underlying computational techniques but also compute different types of organizations such as parallels, rectangles, ellipses, convex groups, and ribbons. However, we are not aware of any general methodology to evaluate such a variety of intermediate level grouping modules. Although there is a continuing debate on the performance characterization of vision algorithms [7] at all levels, most of the demonstrations of the methodologies have been for low level vision modules [8], [9], [10], [11]. Evaluation of complete vision systems has been few [12].

One obvious way to compare organization modules would be to treat grouping as an end in itself and measure its true detection and false alarm rates. However, these measures require the enunciation of ground truth in terms of "true" groups "seen" in the image. For example, one would have to enumerate all possible parallel lines found in an image, preferably, with significance measures. Not only is this task tedious but it is also ill defined.

\footnotetext{
- The authors are with the Department of Computer Science and Engineering, 4202 E. Fowler Ave., ENB 118, University of South Florida, Tampa, FL 33620. E-mail: \{borra,sarkar\}@csee.usf.edu.
}

Manuscript received 2 Aug. 1996; revised 28 Aug. 1997. Recommended for acceptance by $V$. Nalwa.

For information on obtaining reprints of this article, please send e-mail to: tpami@computer.org, and reference IEEECS Log Number 105706.
Consider a set of multiple parallel lines. Does one enumerate all possible pairs of parallels or just the set of "nearest" parallel lines? Clearly, the choice depends on what we are going to use the parallel lines for. Thus, drawing from recent work [7], [8], we believe that performance characterization needs to be done:

1) in the context of a visual task (e.g., object recognition),

2) in a specified domain (e.g., aerial), and

3) it should be done with respect to some ground truth data.

Ideally, one would like to evaluate a grouping module by building a complete object recognition system. However, an artificial recognition system for real domains is still elusive. So, to facilitate the evaluation of grouping modules without building a full recognition system we present five performance measures. These measures can be used to directly predict performance of constrained search based or indexing based recognition. The performance measures are also independent of the organization types and can be used to compare modules producing dissimilar groupings.

Using the performance measures, we evaluate three recent feature grouping modules, namely, the Etemadi et al. [1], Jacobs [2], and Sarkar-Boyer [3] organization modules ${ }^{1}$ on 50 aerial images. We use Analysis of Variance (ANOVA) to guide the experimental design and to statistically analyze the data. ANOVA has been well studied in statistics [13] and used in other experimental fields but its use in computer vision has been limited.

\section{Performance Measures}

In constructing the performance measures, we consider two object recognition strategies: constrained search and indexing. In constrained search we match image features with (2D or 3D) model features under geometric consistency constraints. One way of conducting a constrained search is using a search tree of interpretations. At each level of the tree, we add one new match which is consistent with the matches postulated so far. We conduct a backtracking depth-first search until we completely or at least partially explain the image data. In indexing, a look-up table of model features sets is created off line. At run-time, we simply index into this look-up table using groups of image features, thus, trading space for time.

\subsection{First Measure, $F_{s}^{c}$}

We motivate the first measure from a constrained search recognition point of view. Grimson [4] has derived expressions for the complexity of object recognition using imperfect groups in the presence of clutter. As per Grimson's notation [4], $s$ denotes the number of features in a group, $m$ denotes the number of model features, and $c$ denotes the number of group features which lie on the object or model. The expected search is essentially polynomial if we terminate when the number of matched features equal some predetermined threshold, $t$. The exact expression is given by [4]:

$$
\left.m s \frac{s}{c} \leq W_{t e r m} \leq m t s \frac{s}{c}\left(1+\frac{\kappa^{2}}{m}\right)^{2}\left(\kappa^{2} \frac{s}{m}\right)^{\left\lfloor(s / m) \kappa^{2}-1\right.}\right\rfloor
$$

The constant $\kappa$ is small and is typically equal to $0.2 \frac{P}{D}$ where $P$ is the total perimeter of the object and $D$ is the image dimension. If $\frac{s}{m} \leq 50 \frac{D^{2}}{P^{2}}$, then the search is essentially quartic. In the worst case, $P \approx D$ and the requirement for quartic complexity is essentially $s \leq$ $50 \mathrm{~m}$, a very liberal bound. The term in (1) which depends on the quality of the group is the ratio $\frac{s}{c}$. This constitutes the first performance measure.

1. All the modules are freely available on the internet. We did not "tweak" the codes of these modules for this work. 


$$
F_{s}^{c}=\frac{c}{S}
$$

This measure ranges from zero to one and should be as large as possible to minimize the amount of search.

\subsection{Second Measure, $F_{m}^{c}$}

The quality of the terminated constrained search will be proportional to the threshold, $t$, which is the number of model features explained by the group. Thus, the model to group match quality can be captured by $\frac{t}{m}$. Using this expression coupled with the fact that the termination threshold $t$ is less the number of common features, $c$, between the model and the group, we suggest the second performance measure as,

$$
F_{m}^{c}=\frac{c}{m}
$$

This measure ranges from zero to one and should be large to ensure high confidence recognition. Large values of this measure will help us discriminate between models and thus boost the accuracy of recognition.

\subsection{Third Measure, $F_{m}^{s}$}

Grimson [4] has also shown that the complexity of a constrained search recognition system is polynomial for a complete interpretation if we can assure that all the group features arise from a single object, i.e., there are no spurious features in the group. The third measure is designed to capture this scenario and is of the same form as the second measure but computed for groups which are completely contained in the model.

$$
F_{m}^{s}=\frac{s}{m} \text { if all group features lie on the } \operatorname{model}(c=s)
$$

This measure is also important for indexing. Performance of an indexing system is dependent on the ability of a grouping system to generate "pure" groups with no false data. The sizes of these groups are directly related to indexing speedup $(S)$ which is defined "to be the ratio of the total number of model groups to the number of models found in the index space for each image group" [5]. It can be shown that $a^{s-3} \leq S \leq b^{s-3}$, where $a$ and $b$ are constants and $s$ is the group size [5]. Thus, the larger the group, the better the speedup. The measure $F_{m}^{s}$ captures this group size relative to the model size.

\subsection{Fourth Measure, $F_{d}$}

This performance measure captures the total number of "pure" groups produced, normalized by the number of objects in the scene. A pure group is defined as a group with only object features and no false data.

$$
F_{d}=\frac{N_{\text {true }}^{g}}{N^{m}}
$$

where $N_{\text {true }}^{g}$ is the number of groups which lie completely on an object and $N^{m}$ is the number of objects in the scene. Each pure group is a direct source of partial evidence for a model, be it in a constrained search or indexing framework. With added evidence for a model we expect the confidence in recognition to increase along with the ability to discriminate between models. Thus, ideally, we would like $F_{d}$ to be large for good recognition accuracy.

\subsection{Fifth Measure, $F_{\text {true }}$}

The last measure is the fraction of groups with complete overlap with the object model and is defined as,

$$
F_{\text {true }}=\frac{N_{\text {true }}^{g}}{N^{g}},
$$

where $N_{\text {true }}^{g}$ denotes the number of groups with complete overlap with a model and $N^{g}$ is the total number of groups. The inverse of the measure, $\frac{1}{F_{\text {true }}}$, is the expected number of groups an algorithm needs to generate to find a group which is completely contained in a model. This condition is particularly important for indexing strategies.

For each image, the first two measures $\left(F_{s}^{c}, F_{m}^{c}\right)$ are computed for every group which overlap with an object. The third measure, $F_{m}^{s}$, is computed for groups which are completely contained in an object. Each of these three measures are then averaged over the number of groups (which overlap with an object) to arrive at three values for the whole image. The last two measures are not computed on a per-group basis but are defined over the whole image. Note that the five performance measures can be used to compare algorithms producing different grouping types. The measures provides an indication of how much a grouping module facilitates recognition. Ideally, we would like to maximize the five measures. However, we have to be careful in our interpretation of a head to head comparison of grouping modules. The measures provide judgment on both the choice of the grouping type of an algorithm and the particular computational strategy used.

\section{Creation of Ground Truth}

As we saw in the previous section, the performance measures need the availability of object models, or at least, estimates of the numbers of features $(m)$ in each model. Manual construction of 3D models is cumbersome and renders the performance analysis almost intractable for real domains. We circumvent the problem of actually constructing 3D or 2D models by constructing feature count estimates directly from the images. We manually classify each feature computed from an image into different objects of interest. This classification forms our ground truth. It represents the perfect grouping of features in an image (with no false features in any groups). For 2D model based recognition scenarios such as those that are view based, the number of image features will provide a good estimate of the number of model features. For 3D model based recognition scenarios, we expect the estimates from an image to be proportional to the actual number of $3 \mathrm{D}$ model features (on average).

The evaluated modules group edge segments, hence, our feature type is edge pixel. Thus, $s$ is defined to be the number of edge pixels in a group and $m$ is the number of edge pixels in the manual classification of the image edges. Straight lines or arcs are also possible feature types, however, this would introduce another source of performance variation in the evaluation process, namely, the edge contour segmentation algorithm.

We considered 50 aerial images of airbases, cities, and small forests containing objects such as buildings, hangars, cars, oil tanks, and aircrafts. Fig. 1 shows some examples from the database. On average, each image contains eight objects. We considered three edge detectors to construct the input edge images: Canny [14], Nalwa-Binford [15], and Sarkar-Boyer [16]. All these edge detection algorithms produce single-pixel edges and their implementations are readily available. For each image, we applied all the three edge detectors. The scales for all the three detectors were chosen to be 1.2, and the low- and high-hysteresis thresholds were set at 0.4 and 0.85 , respectively.

For each image, we computed three different types of edges and constructed ground truth classification for each by identifying the pixels belonging to the objects of interest. Figs. $2 b-2 d$ show the ground truth groupings of Canny edges for the gray level image in Fig. 2a. 

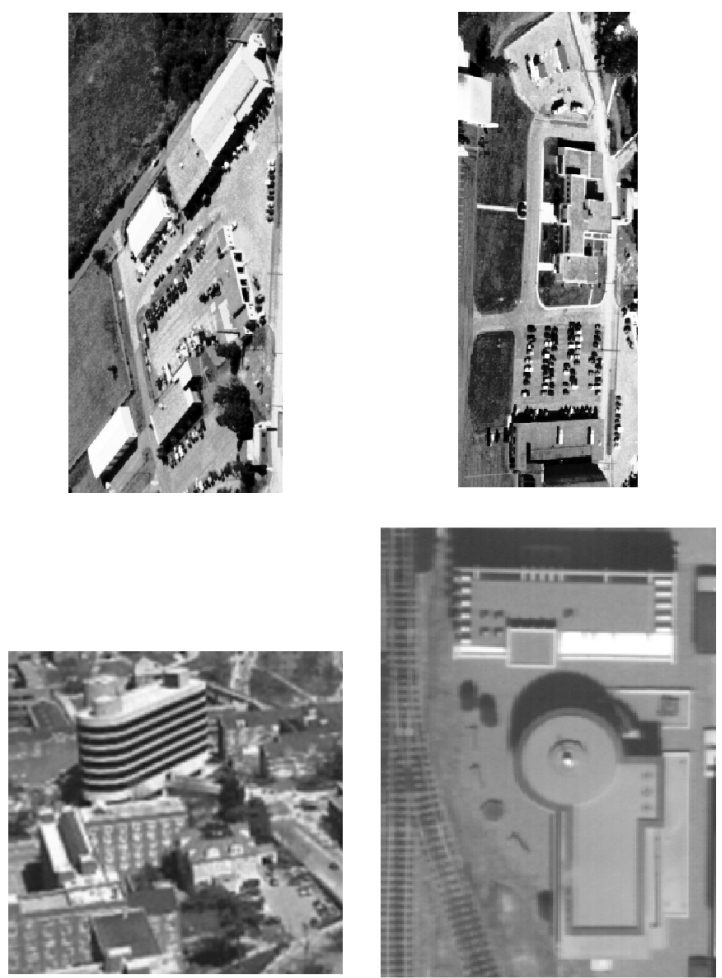

Fig. 1. Some example images from the database of 50 images.

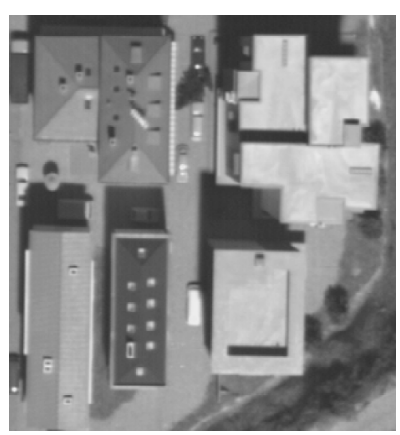

(a)

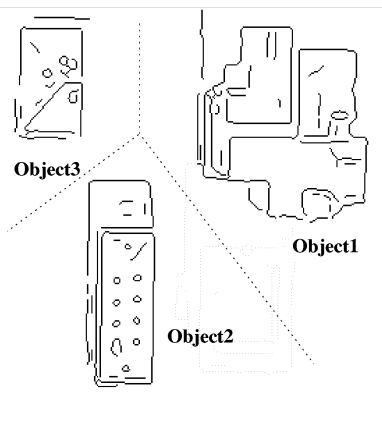

(b)
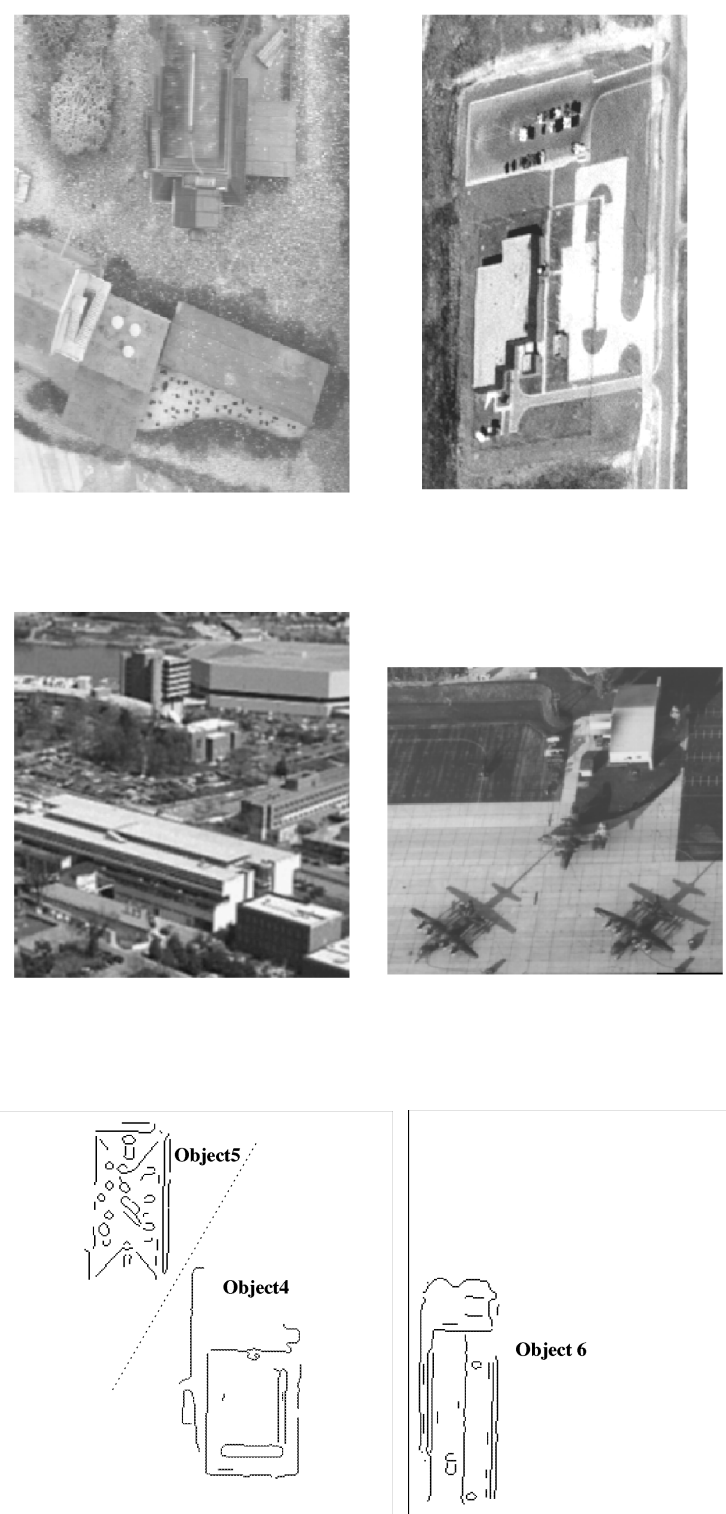

(c) (d)

Fig. 2. (a) An image from the image set. Ground truth groupings of the edge segments found using the Canny edge detector are shown in (b), (c), and (d).

In selecting the edge pixels belonging to an object, we chose edges caused by shadows, depth discontinuity, and significant texture. By significant texture, we mean regular patterns which clearly help in recognizing the object. For example, the stripes of a zebra. Of course, there is always the ambiguity of delineating buildings which are large and complex. Do we have different models for different wings of a building or do we treat the whole building as one? In the present study, we treat such a building as one object. Except for few of these ambiguities, we found it is easy to demarcate the objects of interest.

\section{Modules Compared}

The input to Etemadi et al.'s grouping algorithm [1] is a single pixel edge image. This algorithm first extracts straight lines and then groups them into collinear lines, overlapping parallel lines, non-overlapping parallel lines, T-junctions, V-junctions, Ljunctions, $\lambda$-junctions, triplets of connected segments, $\mathrm{Y}$ and $\mathrm{T}$ Lambda Corners, N-Sided Closed Polygons. Each grouping is associated with a quality factor which captures its significance. This algorithm has four parameters that need to be chosen: the minimum quality tolerance, and the three search pruning parameters, viz., maximum width over height, minimum line length, and minimum $\delta \theta$.

Jacobs [2] proposed an algorithm that locates salient convex collections of line segments in an image. The algorithm finds all convex sets of straight line segments such that the length of the gaps between the consecutive segments is smaller than some fixed proportion of the perimeter. The salient convex groups are found using a constrained backtracking search. The constraint used is a global convexity metric which makes the output of the algorithm reliable and stable. The parameter of choice is one: the saliency threshold $k$.

The Sarkar-Boyer organization module [3] takes edge images as input, computes constant curvature edge segments, and produces groupings which exhibit parallelogram, ribbon, quadrilateral, triangle, circle, and ellipse relationships among them. The Sarkar- 
Boyer algorithm has seven parameters that need to be chosen. They are the center distance, line distance, closure, proximity, orientation and curvature tolerances, and the acceptance distance. These parameters represent the various tolerances that are used to group edge features.

\section{Parameter Selection}

Each organization module has a set of parameters that need to be chosen. The Etemadi et al. module has four parameters of choice. The Jacobs convex grouper has one parameter. And the SarkarBoyer organization modules has seven parameters that can be tuned. We selected a set of 15 parameter combinations for each module in the following manner. For the Etemadi et al. and the Sarkar-Boyer modules we first selected 50 parameter combinations distributed over a reasonable range of parameter values as suggested in the implementation and based on our own experimentation. We applied the modules on five out of the 50 images and noted the subjective performance on a scale of -1 to 1 (definitely not acceptable, acceptable, and surely acceptable). We selected a subset of 15 parameters from this 50 parameter combination set so as to maximize the set of highly acceptable ratings under the constraint that the chosen subset has at least one highly acceptable rating for each of the five images. For the Jacobs module, we selected 15 values of the only parameter over the range experimented with in the paper [2]. For computational reasons we also restricted the maximum number of Jacobs convex groups considered to be 500 per image, a liberal choice given that on average we have eight objects per image.

\section{The Validity of THE EXPerimental SetuP}

We first ask if the experimental design is valid? Do the parameter choices adequately sample the parameter space? Are the images in the data set sufficiently different from each other. To answer these, we computed the five performance measures for every combination of images (50), parameter choices (15), edge detectors (3), and organization modules (3). In all, we had 2,250 runs of each algorithm. We then conducted a statistical ANOVA analysis of the results to interpret the effect of different factors.

Table 1 shows a subset of the ANOVA results ${ }^{2}$ for the three individual organization modules. The first column in each table lists the factors which are potential sources of variations in the measures; they are: edge detectors (E), module parameters $(\mathrm{P})$, image (I), and the pairwise interactions of E, P, and I. For this table, we consider the full parameter set of 15 combinations, three edge detectors, and 50 images. ANOVA divides the total variances in the response amongst various factors. Thus, one might observe that the choice of the edge detector $(\mathrm{E})$, organization parameters $(\mathrm{P})$ and images (I), all contribute to the variance of any organization module output. ANOVA isolates the effect of these individual factors on the response and calculates the significance of the dependence of the response on these factors. These significances are listed second column onwards. The significances measure the probability that the variation due to a particular factor could have arisen due to pure chance. Ideally, this should be less than 0.05 signifying a 95 percent confidence level that the variation is not due to chance. A factor with a significance greater than 0.05 can be considered to have no effect on the variance of the response. Such entries are enclosed in boxes.

Based on the ANOVA results in Table 1, we see that the parameter set for each module (row $\mathrm{P}$ ) is a source of significant variation in the performance. This validates our sampling of the parameter space for each module. The image set also induces a

2. The full ANOVA tables are available at

http://marathon.csee.usf.edu/ sarkar/PO_comparison.html. significant variance in the performance of each of the three modules (see row I of the tables). From an experimental design point of view, this is important. A result to the contrary would suggest a biased image set because it is highly likely that an algorithm would produce different results for different images.

TABLE 1

INDIVIDUAL ANOVA RESULTS FOR THE FULL PARAMETER SET OF 15 COMBINATIONS

\begin{tabular}{|c||c|c|c|c|c|}
\hline \multicolumn{7}{|c|}{ Etemadi et al. } \\
\hline Source & $F_{s}^{c}$ & $F_{m}^{c}$ & $F_{m}^{s}$ & $F_{d}$ & $F_{\text {true }}$ \\
\hline \hline $\mathrm{E}$ & $\mathbf{0 . 0 0 0 1}$ & $\mathbf{0 . 0 0 0 1}$ & 0.0001 & 0.0001 & 0.0001 \\
\hline $\mathrm{P}$ & $\mathbf{0 . 0 0 0 1}$ & $\mathbf{0 . 0 0 0 1}$ & 0.0001 & 0.0001 & 0.0001 \\
\hline $\mathrm{I}$ & $\mathbf{0 . 0 0 0 1}$ & $\mathbf{0 . 0 0 0 1}$ & 0.0001 & 0.0001 & 0.0001 \\
\hline $\mathrm{P} \times \mathrm{I}$ & $\mathbf{0 . 3 6 5 5}$ & $\mathbf{0 . 0 0 0 1}$ & 0.7077 & 0.0001 & 0.0001 \\
\hline $\mathrm{P} \times \mathrm{E}$ & $\mathbf{0 . 0 0 3 7}$ & $\mathbf{0 . 7 8 6 8}$ & 0.2389 & 0.0001 & 0.0008 \\
\hline $\mathrm{E} \times \mathrm{I}$ & $\mathbf{0 . 0 0 0 1}$ & $\mathbf{0 . 0 0 0 1}$ & 0.0001 & 0.0001 & 0.0001 \\
\hline
\end{tabular}

\begin{tabular}{|c||c|c|c|c|c|}
\hline \multicolumn{7}{|c|}{ Jacobs } \\
\hline Source & $F_{s}^{c}$ & $F_{m}^{c}$ & $F_{m}^{s}$ & $F_{d}$ & $F_{\text {true }}$ \\
\hline \hline $\mathrm{E}$ & 0.0680 & 0.0001 & 0.0001 & 0.0001 & 0.0001 \\
\hline $\mathrm{P}$ & 0.0001 & 0.0001 & 0.0001 & 0.0001 & 0.0001 \\
\hline $\mathrm{I}$ & 0.0001 & 0.0001 & 0.0001 & 0.0001 & 0.0001 \\
\hline $\mathrm{P} \times \mathrm{I}$ & 0.0001 & 0.0001 & 0.0001 & 0.0001 & 0.0001 \\
\hline $\mathrm{P} \times \mathrm{E}$ & 0.0001 & 0.0016 & 0.0001 & 0.0001 & 0.9950 \\
\hline $\mathrm{E} \times \mathrm{I}$ & 0.0001 & 0.0001 & 0.0001 & 0.0001 & 0.0001 \\
\hline
\end{tabular}

\begin{tabular}{|c||c|c|c|c|c|}
\hline \multicolumn{7}{|c|}{ Sarkar-Boyer } \\
\hline Source & $F_{s}^{c}$ & $F_{m}^{c}$ & $F_{m}^{s}$ & $F_{d}$ & $F_{\text {true }}$ \\
\hline \hline $\mathrm{E}$ & 0.0001 & 0.0001 & 0.0001 & 0.0001 & 0.0001 \\
\hline $\mathrm{P}$ & 0.0001 & 0.0001 & 0.0120 & 0.0001 & 0.0001 \\
\hline $\mathrm{I}$ & 0.0001 & 0.0001 & 0.0001 & 0.0001 & 0.0001 \\
\hline $\mathrm{P} \times \mathrm{I}$ & 0.0001 & 0.0001 & 0.0731 & 0.0001 & 0.0001 \\
\hline $\mathrm{P} \times \mathrm{E}$ & 0.0501 & 0.9964 & 0.4116 & 0.0001 & 0.0264 \\
\hline $\mathrm{E} \times \mathrm{I}$ & 0.0001 & 0.0001 & 0.0001 & 0.0001 & 0.0001 \\
\hline
\end{tabular}

The boxed entries denote nonsignificant effects under a 95 percent confidence test.

Another interesting observation is that the choice of the edge detector has strong influence on the output of the organization modules (see row E). What is more interesting is that the choice of the edge detector and the images interact (see row $\mathrm{E} \times \mathrm{I}$ ). Thus, the choice of the "best" edge detector, as defined by the performance of the grouping modules, vary with image. This effect needs to be investigated in future.

The $\mathrm{P} \times \mathrm{I}$ rows denote the interaction of the parameter choice and the images. As we see, effect of this factor is significant on all the performance measures except for the first and third measures for the Etemadi et al.'s module and the third measure for the Sarkar-Boyer module. This dependence suggests that the choice of the optimal parameter depend on the image; a fixed parameter choice might not perform equally well on all the images.

\section{Adapted and Fixed Parameters}

Since the parameters and the choice of the edge detector induce significant performance variation, we need to select the "best" edge detector and parameter sets for each module before we can compare any two modules. We selected the best edge detector for each organization module as follows. For every image and 
parameter combination, we ranked the three edge detectors according to each of the performance measures (separately). For each detector, we then summed the ranks over all the image and parameter combinations to arrive at five summed ranks corresponding to the five measures. The edge detector with the smallest sum of these five ranks was chosen as the "best" detector for the organization module. Best results for the Sarkar-Boyer and the Etemadi et al. modules were with the Sarkar-Boyer edge detector. And the Jacobs module performed the best with the Canny detector.

With the chosen edge detector, we then decided on a parameter set for each organization module. There are two possible ways of choosing the optimal parameter set for a module. We can either adapt the parameters on a per image basis (adapted parameter) or choose the parameter combination which results in the best performance over all the images (fixed parameters). We considered both of these. The fixed parameter set for each module was chosen as follows. For each image we ranked the parameter combinations according to each of the five measures (separately). We then summed the ranks over all the images to arrive at five summed ranks for the five measures. The parameter combination with smallest sum of these five ranks is the fixed parameter of choice. The adapted parameter set (one for each image) maximizes the performance on each individual image. For each image, we ranked the 15 parameter values based on each of the five measures. We then summed the five ranks and considered the parameter combination with the best summed rank as the adapted parameter set of choice for that image.

\section{Performance Comparison}

Table 2 shows the distributions of the five performance measures for fixed and adapted parameter sets (with "best" edge detector choices). The columns list the mean values, the 95 percent confidence interval of the means, and the standard deviation of the performance measures. We can use this table of results to compare two modules and also to study the performance of each module with fixed and adapted parameters. However, we have to first establish the statistical significance of the differences.

We conducted ANOVA on each module separately to study the difference between adapted and fixed parameter choices. Partial results are shown in Table 3. Since we fixed the choice of the edge detector for each module, there are just two sources of variation in this study: the image (I) and the parameter choices (P), adapted or fixed. Only the significances for the parameter variable is shown. Images remained to be a significant source of variation for all measures for all modules. As we see in Table 3, the difference in performance between adapted and fixed parameter choices is not significant for only the Etemadi et al. module.

To establish the significance of the observed differences between modules we conducted pairwise ANOVA between all pairs of organization modules. Table 4 lists the significance of the variation due to the modules (row $\mathrm{M}$ ). (The other factor is image (I) which is omitted for lack of space.) The columns represent the module pairs. Note that the significances are computed by ANOVA after accounting for variation due to images. However, we must be cautious when interpreting the significance values since we are performing multiple comparisons. We have to choose the proper significance thresholds. We use the modified form of the Bonferroni test [13]. The threshold significance values for pairwise test, $\alpha_{p}$, is related to overall significance, $\alpha$, by $\alpha_{p}=\frac{(\alpha)(D F)}{n}$, where DF is the degree of freedom and $n$ is the number of comparisons. In our case DF is two, the number of organization modules minus one, and $n$ is three. So, to establish an overall significance of 0.05 (95 percent confidence), we have to test for significance with $\alpha_{p}=0.033$.

We notice that for fixed parameters all pairs of modules have significant differences with respect to all but two measures. The Sarkar-Boyer and Jacobs modules do not differ significantly with respect to the second measure $\left(F_{m}^{c}\right)$. And the Etemadi et al. and Sarkar-Boyer modules do not differ with respect to the fifth measure $\left(F_{\text {true }}\right)$. However, for adapted parameters, the Jacobs and the Sarkar-Boyer module do not differ with respect to three measures $\left(F_{m}^{c}, F_{m}^{s}\right.$, and $\left.F_{d}\right)$. Also, the differences in $F_{d}$ and $F_{\text {true }}$ between the Sarkar-Boyer and Etemadi modules are not significant for adapted parameters. With this in consideration and using the measure estimates listed in Table 2, we can make the observations below.

\subsection{Observation 1}

The Etemadi et al. module does not have significant performance difference between adapted and fixed parameters (Table 3). This reliability is particularly important in practice. It is certainly easier to work with a fixed parameter set than to adapt the parameters of an algorithm on a per-image basis.

The greatest variation between adapted and fixed parameters is for the Jacobs module. This is surprising, especially since the Jacobs module has just one parameter to choose when compared to seven for Sarkar-Boyer and four for Etemadi et al. Although we are not sure exactly why this happens, we feel that it might be because of the nature of the groups generated. For small values of the parameter $k$, Jacobs includes groups with large gaps on the boundary, however, a number of these groups have a large number of common edge segments between them. The essential difference between two groups being sometimes a small segment with size less than the gap being bridged. If this small segment is a part of an object, then its omission from the group will result in lower performance. We need to augment the Jacobs grouper with a post processing step to generate convex groups with the least amount of overlap among them.

\subsection{Observation 2}

The Jacobs module with adapted parameters is the best choice for search based recognition in terms of speed $\left(F_{s}^{c}\right)$ and accuracy $\left(F_{m}^{c}\right.$ and $\left.F_{d}\right)$. However for fixed parameters, although the Jacobs module has the highest $\left(F_{s}^{c}\right)$, Sarkar-Boyer has a better $F_{d}$. The difference in $F_{m}^{c}$ between Sarkar-Boyer and Jacobs modules for fixed parameters is not significant. Thus the confidence in the recognition output with Sarkar-Boyer groups will be the better than Jacobs for fixed parameters.

\subsection{Observation 3}

For fixed-parameter choices, the Sarkar-Boyer module shows the most promise as a good front end for indexing algorithms since it has the best potential speedup $\left(F_{m}^{s}\right)$. However, on adapting the parameters the performance between the Jacobs and Sarkar-Boyer module is indistinguishable.

From the measure $F_{\text {true }}$, we can infer that on average the Jacobs module is more likely to generate groups which lie completely on the object than the Sarkar-Boyer module, both for fixed and adapted parameters. 
TABLE 2

Performance Measures for the Three Organization Modules With Fixed and Adapted Parameter Combinations

\begin{tabular}{|c|c|c|c|}
\hline \multicolumn{4}{|c|}{ Etemadi et al. (Fixed) } \\
\hline & Mean & $95 \%$ & Std. Dev \\
\hline \hline$F_{s}^{c}$ & 0.810 & $0.786-0.834$ & 0.083 \\
\hline$F_{m}^{c}$ & 0.118 & $0.103-0.134$ & 0.055 \\
\hline$F_{m}^{s}$ & 0.129 & $0.110-0.149$ & 0.068 \\
\hline$F_{d}$ & 20.23 & $12.04-28.42$ & 28.82 \\
\hline$F_{\text {true }}$ & 0.658 & $0.623-0.692$ & 0.121 \\
\hline
\end{tabular}

\begin{tabular}{|c|c|c|c|}
\hline \multicolumn{4}{|c|}{ Etemadi et al. (Adapted) } \\
\hline & Mean & $95 \%$ & Std. Dev \\
\hline \hline$F_{s}^{c}$ & 0.811 & $0.787-0.835$ & 0.084 \\
\hline$F_{m}^{c}$ & 0.120 & $0.105-0.136$ & 0.056 \\
\hline$F_{m}^{s}$ & 0.133 & $0.112-0.154$ & 0.075 \\
\hline$F_{d}$ & 23.51 & $8.01-39.00$ & 54.52 \\
\hline$F_{\text {true }}$ & 0.670 & $0.635-0.707$ & 0.122 \\
\hline
\end{tabular}

\begin{tabular}{|c|c|c|c|}
\hline \multicolumn{4}{|c|}{ Jacobs (Fixed) } \\
\hline & Mean & $95 \%$ & Std. Dev \\
\hline \hline$F_{s}^{c}$ & 0.917 & $0.898-0.936$ & 0.066 \\
\hline$F_{m}^{c}$ & 0.163 & $0.135-0.191$ & 0.100 \\
\hline$F_{m}^{s}$ & 0.155 & $0.129-0.180$ & 0.092 \\
\hline$F_{d}$ & 3.929 & $2.465-5.393$ & 5.151 \\
\hline$F_{\text {true }}$ & 0.726 & $0.672-0.781$ & 0.192 \\
\hline
\end{tabular}

\begin{tabular}{|c|c|c|c|}
\hline \multicolumn{4}{|c|}{ Jacobs (Adapted) } \\
\hline & Mean & $95 \%$ & Std. Dev \\
\hline \hline$F_{s}^{c}$ & 0.946 & $0.928-0.964$ & 0.064 \\
\hline$F_{m}^{c}$ & 0.219 & $0.183-0.256$ & 0.129 \\
\hline$F_{m}^{s}$ & 0.216 & $0.180-0.252$ & 0.127 \\
\hline$F_{d}$ & 7.796 & $1.073-14.52$ & 23.66 \\
\hline$F_{\text {true }}$ & 0.796 & $0.735-0.857$ & 0.214 \\
\hline
\end{tabular}

\begin{tabular}{|c|c|c|c|}
\hline \multicolumn{4}{|c|}{ Sarkar-Boyer (Fixed) } \\
\hline & Mean & $95 \%$ & Std. Dev \\
\hline \hline$F_{s}^{c}$ & 0.672 & $0.652-0.691$ & 0.070 \\
\hline$F_{m}^{c}$ & 0.175 & $0.151-0.200$ & 0.085 \\
\hline$F_{m}^{s}$ & 0.200 & $0.166-0.233$ & 0.118 \\
\hline$F_{d}$ & 14.26 & $10.21-18.30$ & 14.23 \\
\hline$F_{\text {true }}$ & 0.649 & $0.605-0.692$ & 0.153 \\
\hline
\end{tabular}

\begin{tabular}{|c|c|c|c|}
\hline \multicolumn{4}{|c|}{ Sarkar-Boyer (Adapted) } \\
\hline & Mean & $95 \%$ & Std. Dev \\
\hline \hline$F_{s}^{c}$ & 0.686 & $0.667-0.705$ & 0.067 \\
\hline$F_{m}^{c}$ & 0.189 & $0.162-0.216$ & 0.095 \\
\hline$F_{m}^{s}$ & 0.217 & $0.180-0.253$ & 0.128 \\
\hline$F_{d}$ & 12.27 & $8.76-15.77$ & 12.33 \\
\hline$F_{\text {true }}$ & 0.660 & $0.616-0.703$ & 0.154 \\
\hline
\end{tabular}

TABLE 3

ANOVA SIGNIFICANCES OF THE DIFFERENCES BETWEEN ADAPTED AND FIXED PARAMETER CHOICES FOR EACH MODULE

\begin{tabular}{|c|c|c|c|c|}
\hline Source & \multicolumn{4}{|c|}{$\operatorname{Pr}>\mathrm{F}$} \\
\hline & Perf. & E et al. & $\mathrm{J}$ & $\mathrm{SB}$ \\
\hline \hline \multirow{3}{*}{$\mathrm{P}$} & $F_{s}^{c}$ & 0.6692 & 0.0042 & 0.0001 \\
\cline { 2 - 5 } & $F_{m}^{c}$ & 0.3261 & 0.0001 & 0.0001 \\
\cline { 2 - 5 } & $F_{m}^{s}$ & 0.0592 & 0.0001 & 0.0001 \\
\cline { 2 - 5 } & $F_{d}$ & 0.4240 & 0.1698 & 0.0005 \\
\cline { 2 - 5 } & $F_{\text {true }}$ & 0.0576 & 0.0074 & 0.0134 \\
\hline
\end{tabular}

\subsection{Observation 4}

The overall performance ranking of the modules is Jacobs, SarkarBoyer, and Etemadi et al. This was obtained by ranking the modules with respect to each measure. For non-significant differences between two modules, both the modules were assigned average of two consecutive ranks. We then summed the ranks of each module over the five measures. For fixed parameters, the total ranks for Jacobs, Sarkar-Boyer, and Etemadi et al. were 8.5, 10, and 11.5, respectively. For adapted parameters, the total ranks for Jacobs, Sarkar-Boyer, and Etemadi et al. were 8, 10, and 12, respectively.

\section{CONCLUSIONS}

In this paper, we proposed five measures and a general experi-

mental framework to characterize perceptual organization or feature grouping modules in the context of object recognition. Although we used these measures to compare three edge based organization modules in the domain of aerial objects, we believe that the methodology is generalizable to groupings of other types of features in different domains. We would also like to draw attention to ANOVA which, as we showed, can provide interesting insights into the performance of a module.

From a grouping module design point of view, in future we need to consider the following. First, is the incorporation of more global cues in the grouping process like that done in the Jacobs convex grouper. This is evidenced by the fact that Etemadi et al. performs worse than Jacobs with respect to $F_{s}^{c}$, even though the former includes groupings of small sizes such parallels and junctions. One might expect that larger fraction of a small group will overlap with an object. However, that is clearly not the case. The Sarkar-Boyer grouper which also has a low performance with respect to $F_{s}^{c}$ is essentially dependent on local search to build the geometric forms. Second, we need to look at more general complex grouping types, e.g., symmetric arrangement of lines, instead of just simple parallel and junction based groupings. The second measure, $F_{m}^{c}$ show that all the three algorithms produce groups which cover about 10 percent to 20 percent of the object; there is much room for improvement. 
TABLE 4

A SUBSET OF ANOVA RESULTS FOR PAIRWISE COMPARISON OF ORGANIZATION MODULES WITH FIXED AND ADAPTED PARAMETERS

\begin{tabular}{|c|c|c|c|c|}
\hline Source & \multicolumn{4}{|c|}{ Pr $>$ F (Fixed) } \\
\hline \multirow{4}{*}{ M } & $\begin{array}{c}\text { Perf. } \\
\text { Measures }\end{array}$ & $\begin{array}{c}\text { SB } \\
\text { E } \text { et al. }\end{array}$ & $\begin{array}{c}\text { E } \text { et al. } \\
\mathrm{J}\end{array}$ & $\begin{array}{c}\mathrm{J} \\
\text { SB }\end{array}$ \\
\hline \multirow{6}{*}{} & $F_{s}^{c}$ & 0.0001 & 0.0001 & 0.0001 \\
\cline { 2 - 5 } & $F_{m}^{c}$ & 0.0001 & 0.0001 & 0.1612 \\
\cline { 2 - 5 } & $F_{m}^{s}$ & 0.0001 & 0.0035 & 0.0001 \\
\cline { 2 - 5 } & $F_{d}$ & 0.0167 & 0.0001 & 0.0001 \\
\cline { 2 - 5 } & $F_{\text {true }}$ & 0.5746 & 0.0080 & 0.0032 \\
\hline
\end{tabular}

\begin{tabular}{|c|c|c|c|c|}
\hline Source & \multicolumn{4}{|c|}{ Pr $>$ F (Adapted) } \\
\hline \multirow{4}{*}{$M$} & $\begin{array}{c}\text { Perf. } \\
\text { Measures }\end{array}$ & $\begin{array}{c}\text { SB } \\
\text { E et al. }\end{array}$ & $\begin{array}{c}\text { E et al. } \\
\text { J }\end{array}$ & $\begin{array}{c}\text { J } \\
\text { SB }\end{array}$ \\
\hline \multirow{6}{*}{} & $F_{s}^{c}$ & 0.0001 & 0.0001 & 0.0001 \\
\cline { 2 - 5 } & $F_{m}^{c}$ & 0.0001 & 0.0001 & 0.0380 \\
\cline { 2 - 5 } & $F_{m}^{s}$ & 0.0001 & 0.0001 & 0.9651 \\
\cline { 2 - 5 } & $F_{d}$ & 0.0802 & 0.0131 & 0.1049 \\
\cline { 2 - 5 } & $F_{\text {true }}$ & 0.5280 & 0.0001 & 0.0001 \\
\hline
\end{tabular}

\section{ACKNOWLEDGMENT}

This work was supported by the U.S. National Science Foundation CAREER grant No. IRI-9501932.

\section{REFERENCES}

[1] A. Etemadi, J.-P. Schmidt, G. Matas, J. Illingworth, and J. Kittler, "Low-Level Grouping of Straight Line Segments," Proc. British Machine Vision Conf., pp. 119-126. New York: Springer-Verlag, 1991.

[2] D.W. Jacobs, "Robust and Efficient Detection of Salient Convex Groups," IEEE Trans. Pattern Analysis and Machine Intelligence, vol. 18, pp. 23-37, Jan. 1996.

[3] S. Sarkar and K.L. Boyer, "Integration, Inference, and Management of Spatial Information Using Bayesian Networks: Perceptual Organization," IEEE Trans. Pattern Analysis and Machine Intelligence, vol. 15, no. 3, pp. 256-274, Mar. 1993. Special Section on Probabilistic Reasoning.

[4] W.E.L. Grimson, "The Combinatorics of Heuristic Search Termination for Object Recognition in Cluttered Environments," IEEE Trans. Pattern Analysis and Machine Intelligence, vol. 13, no. 9, pp. 920-935, Sept. 1991.

[5] D.T. Clemens and D.W. Jacobs, "Space and Time Bounds on Indexing 3D Models From 2D Images," IEEE Trans. Pattern Analysis and Machine Intelligence, vol. 13, no. 10, pp. 1,007-1,017, Oct. 1991.

[6] R. Mohan and R. Nevatia, "Perceptual Organization for Scene Segmentation and Description," IEEE Trans. Pattern Analysis and Machine Intelligence, vol. 14, no. 6, pp. 616-635, June 1992.

[7] R.M. Haralick, L. Cinque, C. Guerra, S. Levialdi, J. Weng, T.S. Huang, P. Meer, Y. Shirai, B.A. Draper, and R. Beveridge, "Performance Characterization in Computer Vision," Computer Vision, Graphics, and Image Processing: Image Understanding, vol. 60, pp. 245-265, Sept. 1994.

[8] T. Kanungo, M.Y. Jaisimha, and R.M. Haralick, "A Methodology for Quantitative Performance Evaluation of Detection Algorithms," IEEE Trans. Image Processing, vol. 4, pp. 1,667-1,674, Dec. 1995.

[9] P.L. Palmer, H. Dabis, and J. Kittler, "A Performance Measure for Boundary Detection Algorithms," Computer Vision and Image Understanding, vol. 63, pp. 476-494, May 1996.

[10] J.L. Barron, D.J. Fleet, S.S. Beauchemin, and T.A. Burkitt, "Performance of Optical Flow Techniques," Proc. IEEE Computer Society Conf. Computer Vision and Pattern Recognition, pp. 236-242, 1992.

[11] R. Zhang, P.S. Tsai, J.E. Cryer, and M. Shah, "Analysis of Shape From Shading Techniques," Proc. IEEE Computer Society Conf. Computer Vision and Pattern Recognition, pp. 377-384, 1994.
[12] D. Nair, A. Mitiche, and J.K. Aggarwal, "On Comparing the Performance of Object Recognition Systems," Int'l Conf. Image Processing, 1995.

[13] G. Keppel, Design of Analysis. Englewood Cliffs, N.J.: Prentice Hall, 1991.

[14] J. Canny, "A Computational Approach to Edge Detection," IEEE Trans. Pattern Analysis and Machine Intelligence, vol. 8, pp. 679-698, Nov. 1986.

[15] V.S. Nalwa and T.O. Binford, "On Detecting Edges," IEEE Trans. Pattern Analysis and Machine Intelligence, vol. 8, pp. 699-714, Nov. 1986.

[16] S. Sarkar and K.L. Boyer, "Optimal Infinite Impulse Response Zero Crossing Based Edge Detectors," Computer Vision, Graphics, and Image Processing: Image Understanding, vol. 54, pp. 224-243, Sept. 1991. 